\title{
EEG based Robot through a BCI Interface for Coal Mines
}

\author{
${ }^{1}$ G. Raghavender, ${ }^{2}$ D. Rupa Kumar, ${ }^{3}$ K.Srinivasa Reddy \\ ${ }^{1}$ M.Tech Scholar, ${ }^{2}$ Associate Professor, ${ }^{3}$ Associate Professor \& HOD ECE \\ ${ }^{1,2,3}$ Department of the Electronics and Communication Engineering, NITS, Hyderabad, TS, India.
}

\begin{abstract}
EEG-based brain-controlled mobile robots will serve as powerful aids for severely disabled individuals in their everyday life, especially to assist them move voluntarily. During this paper, we provide a comprehensive review of the entire systems, key techniques, and analysis problems with brain-controlled mobile robots along side some insights into connected future analysis and development problems. We initial review and classify varied complete systems of brain-controlled mobile robots into 2 classes from the attitude of their operational modes. We tend to then describe key techniques that square measure used in these brain-controlled mobile robots together with the brain-computer interface techniques and shared management techniques. This description is followed by an analysis of the analysis problems with brain-controlled mobile robots together with participants, tasks and environments, and analysis metrics. We tend to conclude this paper with a discussion of the present challenges and future analysis directions.
\end{abstract}

Keywords: BCI, EEG, IEETA, Factors, Brain, Interface, Robot, FMRI, Sensor, MEG, PET

\section{Introduction}

During the last decade several advances in a very variety of fields have supported the thought that an instantaneous interface between the human brain and a synthetic system, referred to as Brain pc Interface (BCI), could be a viable thought, though a big analysis and development effort has got to be conducted before these technologies enter routine use [Berger et al., 2008]. The abstract approach is to model the brain activity variations and map them into some reasonably effort or command over a target output (e.g., a pc interface or a robotic system). Nowadays, the principal reason for the $\mathrm{BCI}$ analysis is that the potential advantages to those with severe motor disabilities, like brain stem stroke, amyotrophic lateral induration or severe brain disorder [Birbaumer et al., 2007; Nijboer et al., 2008; Pfurtscheller et al., 2008]. A very effective thanks to analyze the brain physiological activity is that the graphical record signals stem from the cortex whose sources square measure the action potentials of the nerve cells within the brain.

In this line of thought, the project behind this paper aims to initiate a long-run multidisciplinary analysis by combining developments in relevant fields, like procedure neuro-engineering, signal process, pattern recognition, brain imaging and AI. within the middle-term, the most objective has been the look and development of BCIs to take advantage of the advantages of advanced human-machine interfaces for management and biometrics. This paper presents the advances within the development of $2 \mathrm{BCI}$ systems that analyzes the brain activity of an issue measured through EEG (EEG). the previous tries to search out out the user's intention Associate in Nursingd generates output commands for dominant an applicable output device. The relevant options of the implementation embrace the selection of the motor imagination (mu rhythms) as management paradigm, the efforts dedicated to the event of varied coaching tools and also the user-dependent approach followed. The later explores the likelihood of mistreatment the brain electrical activity throughout visual stimuli for implementing Associate in Nursing graphical record biometric system. Simulation leads to an oversized cluster of subjects indicate the potential of the planned solutions.

The development of interfaces between humans Associate in Nursingd machines has been an increasing field in the last decades. It includes many interfaces mistreatment voice, vision, haptics, diagnostic procedure, electroencephalography (EEG), and mixtures among them as a communication support [1]. A system that analyzes brainwaves to derive info concerning the subjects' status is termed a Brain Computer Interface (BCI) [2]. People who square measure partly or whole paralytic (e.g., by amyotrophic lateral induration (ALS) or brain stem stroke) or produce other severe motor disabilities will realize a BCI as an alternate communicating [3].

In this work, a non- invasive BCI supported graphical record analysis is planned to manage a mobile automaton. management is provided through four specific mental activities: fanciful movements of feet, tongue, left arm, and right arm. These activities square measure correlate with four automaton movements, respectively: stop, move forward, flip left and switch right. The interface classifies the user's mental activity, causing the corresponding command to activate the mobile automaton. Note that the user doesn't ought to be ready to perform such feet, tongue or arm movements, simply imagining them is enough to activate the automaton. 


\section{Framework Of The Analysis}

Over the past decades, many operating BCI systems are delineated within the literature. These systems use a spread of signal acquisition ways, experimental paradigms, pattern recognition approaches and output interfaces, requiring differing types of psychological feature activity [Bashashati et al., 2007; Berger et al., 2008; Ilich Sanchez et al., 2008]. Most solutions have faith in bioelectrical brain signals recorded by graphical record electrodes placed on the scalp. Despite their poor spatial resolution, this non-invasive technique has tried to be a helpful and sensible tool in experimental analysis, primarily as a result of quick recording, simple subject preparation and reduced instrumentation needed. Further, the connection between graphical record and brain perform is well documented within the literature [Niedermayer and Lopes public prosecutor forest, 1999].

In general, a BCI works by analyzing a brain signal, extracting its options and so translating or classifying these options into some recognizable command through the utilization of signal process and machine learning ways. Fig. one illustrates the various useful blocks of a typical EEG-based BCI system. Despite the proof of thought and lots of encouraging results achieved by some analysis teams [Bensch et al., 2007; Leeb et al., 2007; Millán, 2008; Müller-Putz and Pfurtscheller, 2008; Nijboer et al., 2008] further efforts square measure needed so as to make Associate in Nursingd implement an economical BCI. for instance, reliable signal process and pattern recognition techniques ready to unendingly extract substantive info from the terribly howling graphical record continues to be a high challenge.

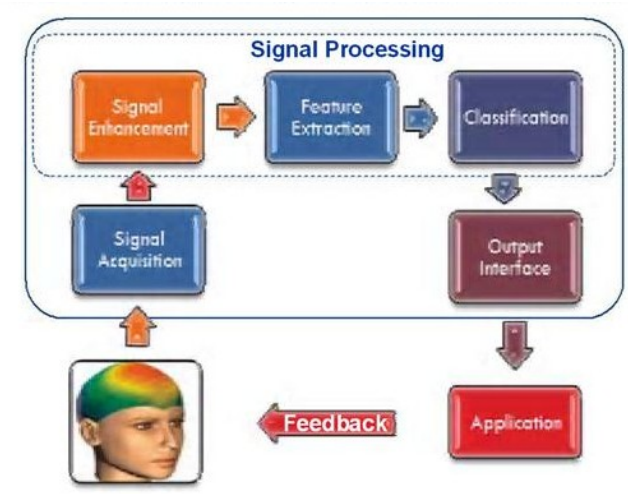

Fig. 1. General components of a BCI system: signals are recorded and enhanced, meaningful features are extracted and subsequently classified, an output signal provides the control command for some target device

The development of noninvasive BCIs for management and biometrics square measure the analysis focus of the IEETA procedure Neuro-engineering analysis cluster and among the foremost recent applications supported personal graphical record knowledge. In spite of sharing a similar basic elements, a BCI to produce an alternate management channel for engaged on the setting and a biometric system for identification or authentication reveal vital variations. whereas the BCI technology has been centered on decoding brain signals for communication and management, needments|the wants|the necessities\} of Associate in Nursing EEG-based biometric system square measure entirely different: they require no interpretation of the brain signals, however the utilization of the maximum amount signal info as doable. The known person is exposed to a information (usually visual or auditory) for an exact time and also the graphical record knowledge collected over this point is input to the biometrics system. it's been shown in previous studies [Paranjape, et al. 1999; Poulos et al. 2001] that the graphical record may be used for building personal identification and authentication systems as a result of the distinctive brain-wave patterns of each individual.

\section{Brain-Computer Interfacing For Control}

This section describes the event steps towards a brain-controlled interface, focusing the issues, challenges and tradeoffs of the entire image. Special attention was given to the planning and analysis of a spread of tools permitting users to adapt and modulate the mu-rythmic activity. Further, the BCI system was designed having in mind a selected user by providing him with a coaching amount within the presence of feedback. As result, this system depends on the particular user management of the brain electrical activity, like amplitude in a very specific waveband (mu rhythms) in graphical record recorded over a selected region (sensoriomotor cortex).

For increased flexibility and flexibility, the developed image relies on transportable and standard ideas supported on MATLAB and Simulink. because of its programming flexibility, all the algorithms area unit written in MATLAB code, whereas the software package algorithmic program for signal acquisition (using a wrapper to integrate it). Another vital facet once developing the system in MATLAB is that it are often tired the next abstraction level, permitting the developer to specialise in the issues of the system and fewer on the tools that support it. Further, many alternative toolboxes offer signal process modules for direct implementation, 
whereas a brand new module are often enforced victimization S-functions to assure the temporal performance essential in on-line operation.

\section{Working Principle}

The principle of operation is kind of easy. 2 dry sensors square measure accustomed find and filter the graphical record signals. The device tip detects electrical signals from the forehead of the brain. At a similar time, the device develop close noise generated by human muscle, computers, light-weight bulbs, electrical sockets and alternative electrical devices. The second device, ear clip, could be a grounds and reference, that permits thinkgear chip to filtrate the electrical noise. The device measures the raw signal, power spectrum (alpha, beta, delta, gamma, theta), attention level, mediation level and blink detection. The raw graphical record knowledge received at a rate of 512 rate. alternative measured values square measure created each second. Therefore, raw graphical record knowledge could be a main supply of knowledge on graphical record signals mistreatment MindWave MW001.

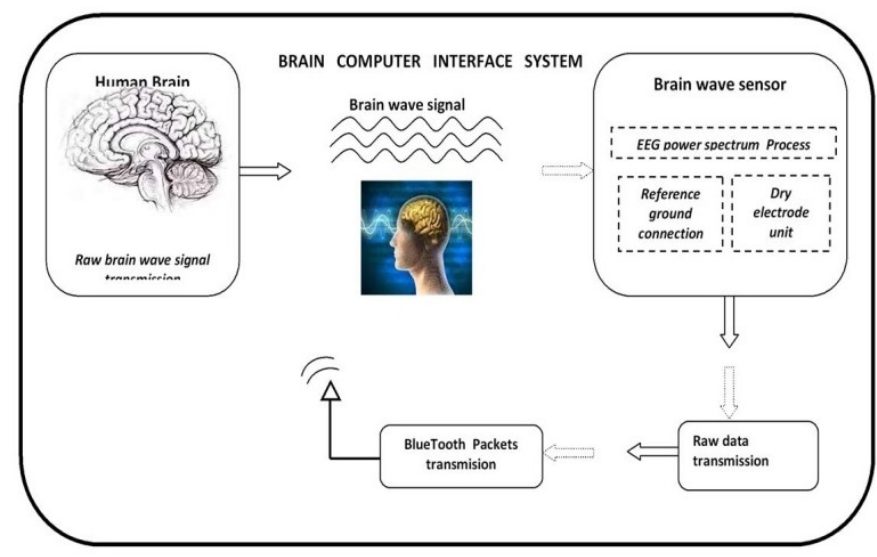

Fig. 2. Brain Computer Interface System

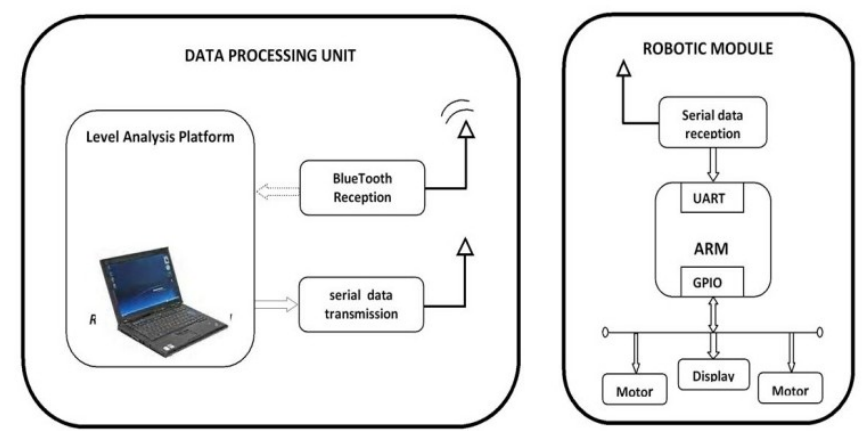

Fig .3. Data Processing unit and Robot Module

\section{Brain Wave Sensor}

In 1924, Hans Berger, a German shrink, performed the primary medical instrument (EEG) recording in humans (Berger, 1929), a discovery that was ab initio greeted with nice skepticism by the scientific community. By recording from one conductor placed over the forehead and one over the bone cortex, Berger discovered the existence of beating activity periodical at close to ten rate, significantly throughout relaxed wakefulness and within the absence of sensory stimulation or mental activity. during this landmark discovery, Berger delineated for the primary time what would become referred to as alpha waves. As a result, Berger was among the primary to counsel that the periodic fluctuations of the human graphical record could also be related to mental processes, as well as arousal, memory, and consciousness. Over the years, developments in knowledge assortment and analyses reworked graphical record into one among the prime techniques for learning the human brain. Table one summarizes elect landmark discoveries and developments that have contributed shaping the graphical record field throughout the century. The past 20 years particularly have witnessed unequalled progress in our ability to image human brain perform non-invasively.

Completely different imaging techniques square measure presently offered to analyze brain perform supported hemodynamic (functional resonance imaging, FMRI), metabolic (positron emission picturing, PET), or magnetism (electroencephalography, EEG; magnetoencephalography, MEG) measurements. so as to analyze spatio-temporal dynamics of brain activity, ways that directly assess neural activity square measure needed. By 
mensuration electrical activity of neuronic assemblies with unit of time temporal resolution, graphical record and one thousand thousand, not like hemodynamic techniques, supply the likelihood of learning brain perform in real time. Sadly, as are going to be mentioned during this chapter, the spatial resolution afforded by EEG/MEG is strained by many factors. the foremost vital of those factors square measure the distorting effects of the top volume conductor, imperfect ratio ratios, and restricted spatial sampling as a result of sensible limits on the numbers of electrodes which will be used. a lot of significantly, it before long became evident that the neuromagnetic "inverse problem" (the conceive to determine generating sources of measured, scalp-recorded graphical record signals) is basically ill-posed. As 1st delineated in 1853 by Hermann von Helmholtz, there square measure Associate in Nursing infinite variety of supply configurations which will justify a given set of scalp-recorded potentials. Thus, at a primary look, the hunt for the event of ways combining unit of time temporal resolution with millimetre spatial resolution seems to be a campaign. as luck would have it, solutions to the inverse downside may be found by postulating physiologically and anatomically sound assumptions concerning reputed graphical record sources and by mathematically implementing established laws of electrodynamics.

\section{Sensors In Coal Mines}

In coal mines for detection of Gases and Metals By using gas sensor, it can detect the harmful gases in mines, wars, industries etc. Whenever a harmful gas is present it will detects by adjusting the sensitivity of the gas sensor, then by indicating the LED. Gas sensors are used in gas leakage detecting equipments in family and industry, are suitable for detecting of LPG, iso-butane, propane, LNG, avoid the noise of alcohol and cooking fumes and cigarette smoke. Resistance value of MQ-6 is difference to various kinds and various concentration gases. So, when using these components, sensitivity adjustment is very necessary. We recommend that you calibrate the detector for 1000ppm of LPG concentration in air and use value of Load resistance ( Rl) about $20 \mathrm{~K} \Omega(10 \mathrm{~K} \Omega$ to $47 \mathrm{~K} \Omega)$. When accurately measuring, the proper alarm point for the gas detector should be determined after considering the temperature and humidity influence. SAR will detect the metallic bombs and dispatches by using metal detector. This circuit detects metal and also magnets. When a magnet is brought close to the $10 \mathrm{mH}$ choke, the output frequency changes. This metal detector project can be powered from a power supply that can provide an output DC voltage between 6 and 12 volt. If a metal is closer to the L1 coil, will produce a change of output oscillation frequency, that will generate a sound in the speaker. Whenever it detects the metallic bombs in the war fields and it dispatch if necessary and it can throw away by using robotic arm.

\section{Experimental Results}

This section describes the experiments allotted so as to verify the effectiveness of the planned solutions. As a primary example, we tend to describe results from Associate in Nursing experiment for the discrimination of 2 mental states: the task is to imagine either right-hand or left-hand movement betting on a visually bestowed cue information within the sort of Associate in Nursing arrow. Second, preliminary results of brain-state estimation mistreatment graphical record signals recorded throughout a self-paced left/right hand movement task square measure bestowed.

An initial effort has been gone in coaching the user to work the system mistreatment the feedback tools delineated on top of. The performance depends on however the user is in a position to attain a state of concentration and learns to properly modulate the mu-rhythms. At a similar time, special attention was dedicated to get the baselines for every hemisphere, as well as tries to pick what baseline offered the simplest results. The notion of an honest baseline is related to the absence of involuntary asynchronism and artifacts. Finally, a style procedure is planned supported the likelihood of mixing graphical record and useful resonance imaging (FMRI) for physiological model standardisation.

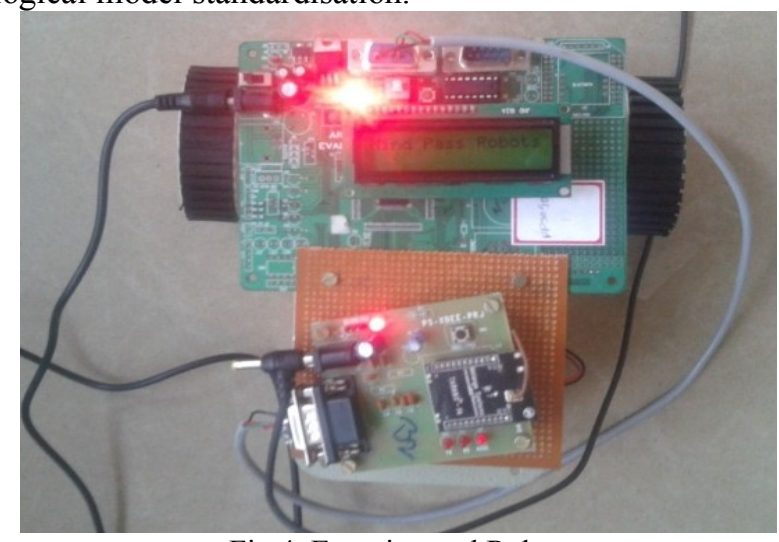

Fig.4. Experimental Robot 


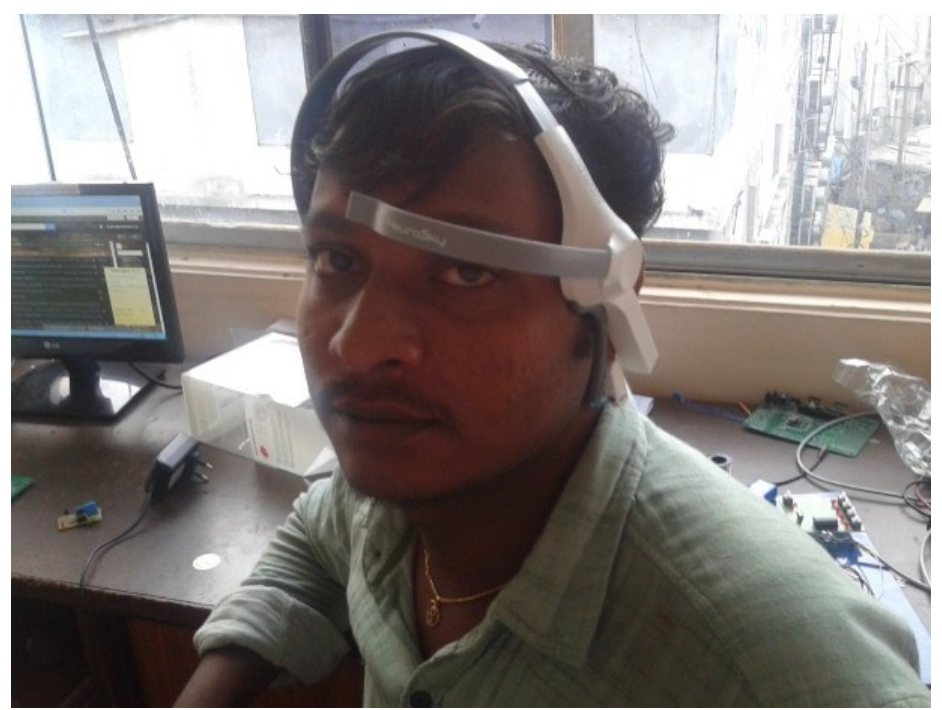

Fig.5. Human with Brain Wave sensor

VIII. Application To A Mobile Robot

To validate the planned methodologies, the developed BCI is applied to a 2-wheeled 120lb mobile automaton. The chosen mobile automaton, named "Touro" (seen in Fig. eight while not its high cover), was already offered within the AI Laboratory from the PUC-Rio University. it had been already programmed to follow radio-frequency (RF) commands, thus no more development was necessary. additionally, such system is Associate in Nursingalogous to an electrical chair, one among the doable applications of the BCI: it's driven by solely 2 active wheels mistreatment differential drive, Associate in Nursingd it's enough traction to hold an adult.

The BCI commands square measure translated to four completely different movements: flip thirty degrees to the proper (RM), flip thirty degrees to the left (LM), move forward 500mm (UM), and stop (DM). Note that any values aside from thirty degrees and $500 \mathrm{~mm}$ can be used.

The communication with the automaton is formed through a PCTx module, that receives values from Associate in Nursing USB affiliation and interprets it into commands to a Futaba $75 \mathrm{MHz}$ RF transmitter that activates the automaton. The laptop portion of the appliance is enforced below the MATLAB ${ }^{\circledR}$ setting, as well as knowledge acquisition from the graphical record $\mathrm{A} / \mathrm{D}$ device, preprocessing, processing, and causing the commands to the PCTx module. The laptop employed in the experiment could be a a pair of. $2 \mathrm{GHz}$ Core a pair of pair notebook. The system activity is performed in 2 steps. within the start, the user has to do mental activities asked by the package, to calibrate it. First, the user is asked to trial (without Associate in Nursingy body movement) an fanciful movement of his/her feet for eleven seconds (a short beep starts the count and an extended beep indicates the tip of the acquisition), from that solely the last 10 seconds square measure recorded. Then, once a 5 second pause, following mental activity (in this case the fanciful movement of his/her tongue) is recorded within the same means, and then on for the left and right arm activities.

\section{Conclusion}

The signal generated by brain was received by the brain device and it'll divide into packets and also the packet information transmitted to wireless medium (blue tooth).the wave unit can receive the brain wave data and it'll convert into signal using MATLAB user interface platform. Then the directions are going to be causation to the house section to work the modules (bulb, fan). The project operated with human brain assumption and also the on off condition of appliance is predicated on dynamic the muscle movement with blinking.

\section{References}

[1]. IEEE-RAS International Conference on Humanoid Robots, pp. 85-90, Daejon, South-Korea, 2008.

[2]. Berger, T.W., J.K. Chapin, G.A. Gerhardt, D.J. McFarland, J.C. Princípe, W.V. Soussou, D.M. Taylor and P.A. Tresco,

[3]. Brain-Computer Interfaces: An International Assessment of Research and Development Trends, Springer, 2008. Birbaumer, N. and L. Cohen, "Brain-computer interfaces (BCI): communication and restoration of movement in paralysis",J. Physiology, January, 2007.

[4]. Fabiani, G.E., D.J., McFarland, J.R., Wolpaw, and G. Pfurtscheller, "Conversion of EEG activity into cursor movement by a braincomputer interface (BCI)", IEEE Transactions on Neural Systems and Rehabilitation Engineering, 12:331-338, 2004. 
[5]. Fatourechi, M., G.E. Birch and R.K. Ward, "A self-paced brain interface system that uses movement related potentials and changes in the power of brain rhythms", J. Computational Neuroscience, 23:21-37, 2007.

[6]. Ferreira A.J.C, EEG-based personal authentication, Master thesis, University of Aveiro, 2009 (in Portuguese).

[7]. Guger, C., A. Schlogl, C. Neuper, D. Walterspacher, T. Strein and G. Pfurtscheller, "Rapid prototyping of an EEG-based braincomputer interface (BCI)", IEEE Transactions on Neural Systems and Rehabilitation Engineering, 9: 49-58, 2002.

[8]. Hoshi, E. and J. Tanji, "Differential involvement of neurons in the dorsal and ventral premotor cortex during processing of visual signals for action planning", J Neurophysiology, 95: 3596-616, 2006.

[9]. C. W. Anderson, "Effects of variations in neural network topology and output averaging on the discrimination of mental tasks from spontaneous EEG," Journal of Intelligent Systems, vol. 7, pp. 165- 190, 1997.

\section{About the authors:}

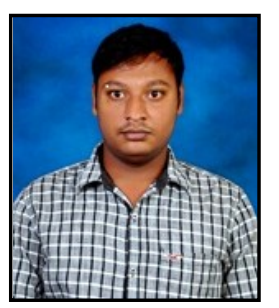

G.RAGHAVENDER I, Pursuing M.Tech in VLSI \& EMBEDDED SYSTEMS at Nagole Institute of Technology \& Science, Hyderabad, Telangana, India. I was received my B.Tech Degree in Electronics \& Communication Engineering is from Sree Dattha Institute of Engineering and Science (SDES), Hyderabad, Telangana, India.

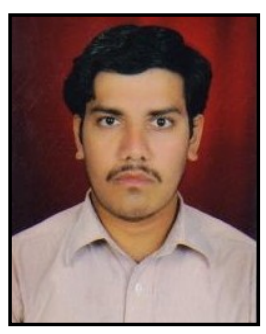

D.RUPA KUMAR is Associate Professor of the Electronics and Communication Engineering, Nagole Institute of Technology and Science, Hyderabad .He received his B.Tech degree in Electronics and Communication Engineering from JNT University, Hyderabad, and M.Tech degree in Vlsi System from JNT University, Hyderabad. He has about 3 publications in National and International Journals.

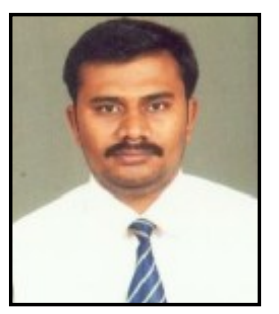

K. SRINIVASA REDDY is Associate Professor of the Electronics and Communication Engineering, Nagole Institute of Technology and Science, Hyderabad .He received his B.Tech degree in Electronics and Communication Engineering from JNT University, Hyderabad, and M.Tech degree in Embedded Systems from JNT University, Hyderabad. $\mathrm{He}$ is a member of The International Association of Engineers (IAENG). He has about 6 publications in National and International Journals. 